\title{
Appareil
}

13 | 2014

Trauma et création

\section{Les vacances du poète}

Daniel Payot

\section{OpenEdition}

Journals

Édition électronique

URL : http://journals.openedition.org/appareil/2010

DOI : 10.4000/appareil.2010

ISSN : 2101-0714

Éditeur

MSH Paris Nord

Référence électronique

Daniel Payot, «Les vacances du poète », Appareil [En ligne], 13 | 2014, mis en ligne le 23 juillet 2014, consulté le 30 juillet 2020. URL : http://journals.openedition.org/appareil/2010 ; DOI : https://doi.org/ 10.4000/appareil.2010

Ce document a été généré automatiquement le 30 juillet 2020.

\section{(c) (i) () $\Theta$}

Appareil est mis à disposition selon les termes de la Licence Creative Commons Attribution - Pas d'Utilisation Commerciale - Pas de Modification 4.0 International. 


\title{
Les vacances du poète
}

\author{
Daniel Payot
}

\section{Athènes, Alexandrie, Rome}

1 Ça commence comme une puissance fabuleuse : «tout ce qui est cause du passage du non-être vers l'être pour quoi que ce soit, voilà en quoi consiste la fabrication (poiésis) ${ }^{1}$ ». Mesure-t-on bien la portée d'un tel «passage »? Du non-être vers l'être, ce n'est pas anodin... Et cela concerne "quoi que ce soit»: tout ce qui n'est pas rien et qui ne pousse pas tout seul.

2 Les « ouvrages réalisés par tous les arts » sont certainement, dans leur composition intrinsèque et quoi qu'ils exposent, des célébrations de cette puissance, puisque ce sont des «fabrications (poieseis)». Et ceux qui les réalisent en sont certainement des servants, puisque ce sont des «fabricants (poietai) ${ }^{2} »$. Ce sont des passeurs, cerbères inverses, Charon à rebours, nochers à contresens. Les poètes n'accompagnent pas les âmes vers le royaume d'Hadès, ils guident au contraire ce qui n'était pas, et qui maintenant devient, vers la lumière. Dès que pointe l'artefact, un Orphée le hisse.

Le pouvoir de ces convoyeurs ascensionnels est extraordinaire, étrange, inquiétant. Ne faut-il pas posséder un savoir singulier du non-être pour parvenir ainsi, parfois, à l'abolir? Ce savoir ne témoigne-t-il pas d'une particulière affinité avec les lieux dans lesquels les non-encore-choses, inertes, tapies, impatientes peut-être, attendent l'arrivée des passeurs? Et n'y faut-il pas aussi l'acuité, la cruauté, l'inflexible souveraineté de qui choisit, parmi toutes ces désirantes, celles qui seules seront?

4 Ce qui chez le poète est idéalisation, désir d'embellissement, dispensation prodigue provient d'une accointance avec ce qui s'agite, se promet, exulte dans l'espace infiniment inhospitalier de l'absence. L'abondance n'est qu'une ruse; sous elle, lui donnant lieu, il y a le sens acéré, l'intelligence, la maîtrise de ce qui n'est pas. Les artistes "suppléent au défaut des choses ", écrit Plotin ${ }^{3}$. Le supplément n'est pas une augmentation de l'existant, c'est un commerce avec l'inexistant. Phidias, ajoute-t-il, «fit son Zeus, sans égard à aucun modèle sensible; il l'imagina tel qu'il serait, s'il 
consentait à paraître à nos regards ». D'où vient au poiète ce discernement, cette emprise du conditionnel ? D'où ce savoir? D'où ce pouvoir?

5 La poiésis, Aristote la définissait comme « œuvre extérieure à l'artiste $»^{4}$. La distance, la non-coïncidence, la différance du fabricant et de la fabrication ne pourraient-elles être ce qui reste, en la marquant, en en inscrivant la trace dans la place de l'œuvre, de l'affinité du poète avec le non-être depuis lequel il la fait venir? Elles désigneraient alors une vacance constitutive dans la fabrication même.

\section{Saint-Michel-de-Montaigne, Ménilmontant}

6 Que la capacité de hisser vers l'être soit aussi l'effet d'un singulier acoquinement avec un monde distant, distendu, peuplé de « choses » qui n'en sont pas, qui en tout cas ne sont pas articulées aux nôtres et échappent à nos continuités et à nos enchaînements, c'est peut-être ce qui, pour quelques Européens notoires, constitue la signification de l'accident, et particulièrement de la chute. Cette épreuve les précipite dans une contrée dont ils ne connaissent ni ne reconnaissent rien; ils en ramènent, s'ils survivent, des expériences et des phénomènes inédits qu'ils savent ensuite intégrer dans les continuités et dans les enchaînements de leur monde manifeste.

7 Entre le choc violent avec le "puissant roussin » monté par l'un de ses gens " grand et fort » et l'enseignement qu'il dégage de son évanouissement consécutif, Montaigne tient à consigner les épisodes et séquences intermédiaires ${ }^{5}$ : le coma, le transport, le semi-réveil et les vomissements de sang, les premières sensations et premiers "pensements» (pourquoi n'a-t-on pas gardé ce terme si suggestif dans l'usage moderne de la langue ?), les réactions et émotions des proches, les perceptions insensibles et les douleurs physiques. La consécution est méticuleusement décrite. D'abord, la mort : « voilà le cheval abattu et couché tout étourdi, moi dix ou douze pas au-delà, mort, étendu à la renverse, le visage tout meurtri [...], n'ayant ni mouvement, ni sentiment, non plus qu'une souche». Puis le retour des "premiers sentiments [...] beaucoup plus approchants de la mort que de la vie». Montaigne reprend un peu ses esprits et commence à parler, mais « je n'y étais aucunement ; c'étaient des pensements vains, en nuë », qui «ne venaient pas de chez moi », puisque je ne savais «ni d'où je venais, ni où j'allais"; "ce que l'âme y prêtait, c'était en songe, touchée bien légèrement, et comme léchée seulement et arrosée par la molle impression des sens ». La séquence suivante est très positive : «mon assiette était à la vérité très douce et paisible; je n'avais affliction ni pour autrui ni pour moi ; c'était une langueur et une extrême faiblesse, sans aucune douleur. Je vis ma maison sans la reconnaître. Quand on m'eut couché, je sentis une infinie douceur à ce repos »; "C'eût été sans mentir une mort bien heureuse ; car la faiblesse de mon discours me gardait d'en rien juger, et celle du corps d'en rien sentir. Je me laissai couler si doucement, et d'une façon si molle et si aisée, que je ne sens guère autre action moins poisante que celle-là était ». Mais deux ou trois heures après, et pendant plusieurs jours, ce sont douleurs physiques et évocation d'une mort qui ne serait plus tranquille coulée, mais violence affilée. Enfin, avant-dernière étape, le souvenir: "quand ma mémoire vint à s'entr'ouvrir, et me représenter l'état où je m'étais trouvé en l'instant que j'avais aperçu ce cheval fondant sur moi [...] il me sembla que c'était un éclair qui me frappait l'âme de secousse, et que je revenais de l'autre monde». Et pour finir, la morale de l'épisode tout entier : «à la vérité pour s'apprivoiser à la mort, je trouve qu'il n'y a que de s'en avoisiner ». 
8 La formulation de l'adage ne peut être dissociée des lignes qui le précèdent et lui donnent son épaisseur : c'est dans la succession des états décrits que l'instruction et le sens se fabriquent. Or ces états ne sont que des stations dans une marche continue, des arrêts sur image d'un déroulement, les détails d'un mouvement que seule la narration peut suggérer. Comme toujours, Montaigne peint le passage, et pas l'être ${ }^{6}$. Ici, c'est particulièrement vrai : passage de la mort à la vie, retour d'une excursion dans l'autre monde, ascension d'un Mont Ventoux qui ne serait autre que le lieu d'habitation des vivants. Pétrarque faisait monter Orphée jusqu'aux cieux chrétiens, Montaigne fait revenir l'un et l'autre dans la simple familiarité d'un quotidien qui serait inepte si les mortels qui l'habitent ne savaient se familiariser aussi, par l'expérience, avec ce qui les attend. Ils y vont, le meilleur exercice pour y consentir est d'en venir. Et en venir, c'est se souvenir, retrouver la mémoire de l'événement qui a causé une faille irréparable, un moment d'absence, un usage du non-être. L'apprivoisement suppose le voisinage, mais c'est le voisinage avec ce qui ne saurait être apprivoisé, ni même imaginé : l'absence. Celle-ci demeure patente dans les pas du retour: «je n'y étais aucunement». Maintenant, je marche de nouveau, mais ma marche est scandée par ces intervalles de non-être en elle, par ces blancs qui écartent d'elle-même l'opacité aveugle d'une vie sans percée, sans traversée. S'il n'y avait la douleur physique, cette disposition serait des plus heureuses : ne plus savoir, ne plus reconnaitre, ne plus ressentir d'affliction, se laisser seulement porter dans la coulée du songe, dans les allers et retours de la barque du nocher, dans l'incessant traghetto qui relie être et non-être, cela constituerait les meilleurs conditions qui soient à la fabrication de la sagesse. Le choc des chevaux a ouvert une poétique de l'acquiescement liquide, une poétique des rives raccordées.

Chez Rousseau, les chevaux ne sont pas loin, mais c'est un "gros chien danois » qui renverse le fabricateur de concepts et de confessions revenant de sa promenade sur les hauteurs de Ménilmontant ${ }^{7}$. Le choc est violent, la tête plus lourde que les pieds, le pavé «très raboteux ». Le récit du réveil n'est pas aussi détaillé que chez Montaigne, mais tout y est, le sang et le plaisir, la sensation et l'oubli, les délices d'une présence au monde et à soi que n'altère nulle médiation, nul intérêt, nulle compromission.

Ce retour est un "moment délicieux ", l'expérience inouïe d'assister à sa propre naissance, de vivre ce passage par les seuls effets du sentir, sans notion, sans idée, sans conscience et donc sans crainte ni inquiétude. Une pure esthétique, antérieure au bien et au mal comme à toute distinction entre subjectivité et objectivité, une heureuse affection antérieure à tout savoir. Il ne s'agit pas vraiment de la disparition de toute chose, du retrait de tout vis-à-vis. Ce n'est pas comme si le monde s'absentait et laissait au sujet la chance d'une affirmation suffisante de soi, c'est une tout autre relation qui advient entre le sujet et lui-même, faite d'adéquation et d'absentement simultanés : «Tout entier au moment présent je ne me souvenais de rien ». L'expérience est celle d'une abstraction temporelle, d'une suspension de toute chronologie au profit du seul instant. Et pourtant, cet instant exclusif n'est pas adéquation à soi du sujet, n'est pas plénitude, mais au contraire effacement: "je n'avais nulle notion distincte de mon individu, pas la moindre idée de ce qui venait de m'arriver ; je ne savais ni qui j'étais ni où j'étais ». Il est manifestement délicieux de se sentir sans se savoir; d'être là sans avoir à construire une représentation ni de soi, ni du là. D'être présent sans médiation. C'est une définition de la jouissance: "Je sentais dans tout mon être un calme ravissant, auquel chaque fois que je me le rappelle, je ne trouvais rien de comparable dans toute l'activité des plaisirs connus ». 
11 La suspension de toute intercession transforme le rapport à soi en ravissement. Elle n'est pas exclusive d'une relation au monde, mais celle-ci devient pure poétique. Se substituant à la lourdeur de la perception, de la réception, de l'appréhension de la réalité extérieure, l'expérience est celle d'une projection: «il me semblait que je remplissais de ma légère existence tous les objets que j'apercevais ». Ces objets ne sont plus opaques, ils ne forment plus des obstacles, ils ne s'opposent plus à ma progression. Ils s'ouvrent, au contraire, pour m'accueillir, pour m'héberger. Mais que puis-je leur confier, que puis-je projeter en eux, si je ne suis plus moi-même qu'évanescente affection, que subtil passage dénué d'attestation de soi? Qu'ai-je encore en moi qui puisse «remplir» ces objets? Ce qu'il reste, que Rousseau nomme «ma légère existence », n'est ni principe, ni substance, mais l'acte même du venir, de l'émergence, de la naissance. Je ne remplis pas les choses d'un "moi-même " qui serait lourde matière ou profonde pensée, mais d'un moi allégé de tout rapport à soi, de toute représentation, de toute valeur. Est-ce que les choses en seront alors transformées? Nullement, puisque le mouvement est si délicat qu'aucun effet physique ne peut en être attendu. Mais oui, complètement, puisque, emplies de ma légèreté, les choses vont être émancipées de leur propre pesanteur, de leur propre adéquation à soi. Et c'est le commencement de la poésie : l'évanouissement a transformé le sujet en poiète, sa fabrication d'écriture transforme les choses en matière de songe. La rêverie de ce promeneur-là, déjà, a «transposé un fait de nature en sa presque disparition vibratoire", et toute fleur aperçue près des pavés raboteux du chemin de Ménilmontant est déjà une espèce $d^{\prime}$ " absente de tout bouquet ${ }^{8}$ ».

12 Au réveil, on se consacre, selon les mots choisis par Mallarmé dans le même passage, à « un art consacré aux fictions »- sans doute parce qu'il est alors devenu désirable de faire quelque chose de la légèreté acquise de son existence, d'emplir le monde du bonheur d'avoir été présent dans une absence à soi.

\section{Paris, avenue du Bois}

13 Des pavés, « inégaux » et « mal équarris » plus que raboteux, il y en a aussi dans la cour de l'hôtel que le Prince de Guermantes vient de se faire construire à Paris, avenue du Bois. Marcel, épuisé et au comble de l'abattement, s'y rend à une matinée mondaine et musicale dont il doute d'avance de l'efficacité consolatrice. Ce qui se passe alors constitue pour la littérature de langue française l'un des épisodes les plus considérables.

En roulant les tristes pensées que je disais il y a un instant, j'étais entré dans la cour de l'hôtel de Guermantes, et dans ma distraction je n'avais pas vu une voiture qui s'avançait ; au cri du wattman je n'eus que le temps de me ranger vivement de côté, et je reculai assez pour buter malgré moi contre les pavés assez mal équarris derrière lesquels était une remise. Mais au moment où, me remettant d'aplomb, je posai mon pied sur un pavé qui était un peu moins élevé que le précédent, tout mon découragement s'évanouit [...].

La séquence qui commence par ces deux phrases est celle où se décide l'écriture de La Recherche : entre l'accident évité de peu et la détermination d'une poétique singulière, le lien est explicite et longuement développé, dans des pages où l'on voit le livre instituer son propre commencement, relater sa propre naissance, circonscrire la brèche dans laquelle il inscrit, pour se l'approprier, sa propre genèse ${ }^{9}$. 

propre puissance, puisque son origine même s'y trouve racontée, puisqu'il rend compte de son aptitude à énoncer de lui-même son propre passage du non-être à l'être. Mais cette puissance poiétique est en même temps l'aveu d'une déchirure constitutive et de l'imminence constante d'un absentement, comme si le projet d'une exhibition par soi de sa propre origine, en cela jamais achevable ni même vraiment possible, conduisait son auteur au plus près de la disparition. d'azur. Aussitôt le danger écarté, l'inégalité des pavés de la cour de l'hôtel des Guermantes devient celle des dalles du baptistère de Saint-Marc à Venise. L'événement est identique à celui qui faisait que la petite madeleine contenait tout Combray. Bientôt, le choc d'une cuiller contre une assiette restituera le souvenir heureux d'un train arrêté dans un petit bois, et le contact de la serviette avec laquelle l'auteur s'essuie la bouche rendra de nouveau présente la plage de Balbec. Marcel attend dans le salonbibliothèque de l'hôtel que se termine le morceau de musique en cours, « la princesse ayant défendu qu'on ouvrît les portes pendant son exécution », et il se laisse envahir par le bonheur merveilleux de ces associations. Des visions remontent, des sensations, jointes les unes aux autres, sont redonnées, qui étaient « restées dans l'attente, à leur rang, d'où un brusque hasard les avaient impérieusement fait sortir, dans la série des jours oubliés » (p. 867).

17 Chacune est à la fois, indissociablement, «le miracle d'une analogie » (p. 871) et la chance d'une « résurrection ${ }^{10} »$ du moi. Chacune signifie l'ouverture, à l'occasion d'une rencontre impromptue ou d'un choc, d'un de ces vases conservés en nous, «disposés sur toute la hauteur de nos années", "situés à des altitudes bien diverses", qui contiennent, avec les paroles dites, avec les gestes accomplis, le reflet des choses qui les entouraient, les couleurs, les odeurs, les températures qui constituaient leurs conditions et leur environnement. Ces vases sont hermétiques, ils n'ont rien perdu de ces atmosphères, et quand l'un libère son contenu, ce qui se diffuse n'est pas seulement la chose ou la situation que l'on se rappelle, mais le monde tout entier dans lequel celleci baignait.

Pour que cela advienne, il faut qu'un lien s'établisse, qu'une agrafe assemble le moment actuel et le moment éloigné, le présent et le passé. En un éclair, les deux modalités du temps se superposent, empiètent l'une sur l'autre, se fondent l'une dans l'autre. Cet éclair déchire le temps, il n'est lui-même réductible à aucun mode temporel, il fait scintiller soudain le site miraculeux de l'extra-temporel. La fusion du présent et du passé n'est ni présent, ni passé, elle est, fugitivement, dans l'instantanéité de la sensation, l'événement pur, libéré du poids de la temporalité. La durée de l'éclair est celle de l'advenue de «l'essence des choses» (p. 871, p. 872), soudain émancipées de leur contingence ; elle est aussi celle de la jouissance du moi vivant enfin cette essence. Elle lui permet « d'obtenir, d'isoler, d'immobiliser [...] ce qu'il n'appréhende jamais : un peu de temps à l'état pur » (p. 872).

19 Sentir le temps «à l'état pur », pour le moi, c'est le vivre tel qu'en lui-même, dans son essence qui en soi ne dépend d'aucune temporalité : cette essence n'est précisément aucune chose dans le temps, elle est temps même, idée du temps en tant que telle soustraite au temps subi comme condition et contingence. C'est pourquoi le souvenir n'est pas un voyage dans le temps. Retrouver « les jours anciens, le temps perdu, devant qui les efforts de ma mémoire et de mon intelligence échouaient toujours » (p. 871), ce 
n'est pas pour le moi se transposer du moment actuel en un moment antérieur, c'est sortir du temps, et c'est seulement quand le moi accède pour un instant à cet en-dehors qu'il peut vivre vraiment, absolument, ce qui lui est alors restitué : Venise, Combray, Balbec, non comme des conditions spatio-temporelles, mais comme des condensés d'éternité. Dans la cour de l'hôtel de Guermantes, Marcel ne se remémore pas Venise, il n'en construit pas une représentation, il y est, il se trouve corps et âme dans une Venise qui serait certes une sorte d'absente de toute lagune, et qui pourtant est l'assomption de Venise, la vérité de sa présence.

Avec le miracle de l'analogie advient aussi la renaissance, la résurrection du moi. L'événement porte en lui "l'idée d'existence » (p. 872), et le moi, d'habitude empêtré dans les aléas de la spatialité et de la temporalité, d'ordinaire aliéné dans la contingence du monde, est redonné à lui-même au moment où lui est donnée l'essence glorieuse de ce qui fait retour en lui.

Mais qu'un bruit, qu'une odeur, déjà entendu ou respirée jadis, le soient de nouveau, à la fois dans le présent et dans le passé réels sans être actuels, idéaux sans être abstraits, aussitôt l'essence permanente et habituellement cachée des choses se trouve libérée, et notre vrai moi qui, parfois depuis longtemps, semblait mort, mais ne l'était pas entièrement, s'éveille, s'anime en recevant la céleste nourriture qui lui est apportée. Une minute affranchie de l'ordre du temps a recréé en nous, pour la sentir, l'homme affranchi de l'ordre du temps. (p. 872-873)

21 La crise aiguë précédant la décision d'écrire le livre possède ainsi une dimension qu'on pourrait appeler phénoménologique: suspension des apparences mondaines immédiates, mise entre parenthèses des prérogatives de l'intelligence et de la volonté, épochè et réduction eidétique, dégagement des essences. Parfois d'ailleurs l'écriture même de la séquence, sa progression, son côté méthodique pourraient évoquer quelques Méditations cartésiennes, voire quelques Méditations métaphysiques. Avec, à titre de motif accentué et de finalité d'ensemble, une forme d'ontologie du moi : l'ambition d'accéder à la vérité vivante, à ce qui constitue l'être de l'homme d'habitude étouffé sous les organisations spatio-temporelles du monde.

Mais quand il atteint cette strate originelle, le texte rencontre une autre dimension encore, dont le premier effet est de différer, peut-être à l'infini, la résolution de la crise que promettait son inspiration métaphysique (les essences, la transcendance du pur par-delà la temporalité, l'avènement de la vérité par-delà la finitude mondaine). Proust écrira La Recherche, et non Matière et Mémoire, Leçons pour une phénoménologie de la conscience intime du temps ou Être et Temps. Et La Recherche, malgré le titre de son dernier volume, Le Temps retrouvé, qui ne contient ni réappropriation générale, ni achèvement systématique, est plus que tout le récit d'une crise interminable, que ne vient interrompre nulle résolution et nulle thèse. C'est pourquoi le moment du livre que nous sommes en train de lire, où celui-ci fait retour sur sa propre genèse, est à ce point décisif : à titre de vérité accomplie, Proust ne nous y donne en fait que l'indication de la nécessité du récit lui-même, sans terme ultime où son mouvement viendrait échouer et s'exhausser, le mouvement immanent du récit étant sa seule vérité effective.

Marcel s'efforce de comprendre ce qui différencie le souvenir donné dans la soudaineté accidentelle de l'éclair et la mémoire volontaire et consciente. Il note que cette dernière ne fait "que combiner entre eux des éléments homogènes" et que les souvenirs qui viennent de l'assaillir (Venise, le train arrêté dans le petit bois, la plage de Balbec) ne procèdent pas d'une association de ce type. L'éclair et la cristallisation qu'il produit, le choc de la rencontre d'un moment du présent et d'un moment du 
passé, leur empiètement fortuit, ne sont en rien comparables à un agencement dans l'homogène, ni à une réduction des deux éléments à l'unité d'une même condition. L'expérience n'est précisément pas celle d'une conjonction dans l'identité ou dans la ressemblance, mais d'un court-circuit disjonctif, d'une incandescence extraordinaire n'appartenant à aucun des éléments mis en contact et faisant miraculeusement surgir une vérité tout autre. Quand Marcel ajoute alors: «dans les trois souvenirs que je venais d'avoir [...], au lieu de me faire une idée plus flatteuse de mon moi, j'avais, au contraire, presque douté de la réalité actuelle de ce moi»(p.873), il construit un rapport explicite entre l'image valorisée du moi et son pouvoir de combiner dans l'homogène et il suggère au contraire la fragilité du moi soudain jeté dans la tempête de l'événement, sous le coup des éclairs et des cristallisations fortuites. Le moi n'assure plus alors une fonction d'ordonnateur et de metteur en œuvre, il ne maîtrise plus la situation depuis l'éminence ou le surplomb de qui sait se saisir des choses, les manipuler, les associer plus ou moins judicieusement les unes aux autres. Dans l'éclair, la place du moi cesse d'être évidente, et la crise poétique que vit Marcel, jusque dans sa joie manifeste, est directement celle d'une déstabilisation, d'une destitution, d'une déposition. Le "doute " qu'il évoque rejoint des indications qu'on découvrait un peu plus haut, quand il était question de «l'ébranlement effectif de mes sens» (p. 872) et d'un empiètement du passé et du présent susceptible de « me faire hésiter à savoir dans lequel des deux je me trouvais » (p. 871).

C'est que, "entre le souvenir qui nous revient brusquement et notre état actuel, de même qu'entre deux souvenirs d'années, de lieux, d'heures différentes, la distance est telle que cela suffirait [...] à les rendre incomparables les uns aux autres» (p. 870). Tout tient à cela, à cette distance incommensurable et au fait qu'elle ne peut être abolie par la volonté, l'intelligence ni le savoir-faire agenceur du moi. Si deux états se trouvent en un éclair associés, leur conjonction est donc l'effet d'une autre puissance que celle du moi. Cette puissance est celle qui a perçu et enregistré les choses et les atmosphères, les a enfermées dans des vases, a préservé l'étanchéité de ces vases, a consenti soudain à leur libération. Elle ne porte pas dans le texte de nom unique, peut-être parce que son identification excède les limites d'un moi qui ne tiendrait à l'inverse que d'elle sa capacité de nommer. Mais elle est approchée par les effets conjugués de plusieurs vocables et expressions.

Elle est ainsi, d'abord, une puissance d'oubli. C'est « grâce à l'oubli » que le souvenir est resté intact dans son vase hermétique, c'est grâce à lui qu'a été perdu ce qui aujourd'hui miraculeusement revient, dans le halo de « cet air plus pur que les poètes ont vainement essayé de faire régner dans le Paradis", tentative chimérique tant qu'elle ne comprend pas que "les vrais paradis sont les paradis qu'on a perdus" (p. 870). Puissance de perte, donc, qui dans le texte bénéficie du même retournement que l'oubli et la distance: ces termes d'abord négativement connotés en viennent à nommer, positivement, ce qui permet le souvenir et son bonheur insigne. La joie ne viendrait pas si les moments et les éléments associés ne l'étaient pas depuis leur éloignement irréductible, depuis l'obscurité de leur enfouissement, depuis l'opacité de leur non-être.

Plus le texte souligne la perte, plus il souligne aussi le caractère involontaire et inexpliqué du renouvellement, de l'exhumation et du passage à la lumière. La perte, la distance sont telles, que le souvenir devrait être, à la lettre, impossible. S'il survient pourtant, c'est par l'effet d'un "expédient merveilleux de la nature » (p. 872), d'un 
«subterfuge» (p. 872), d'un «trompe l'œil » (p. 873), termes ambigus qui ajoutent à la destitution relative du moi, qui se trouve donc à la fois victime (comme on l'est d'une illusion) et bénéficiaire (dans sa joie soudaine) d'une effectivité qu'il ne contrôle en rien.

Cette position ambivalente est encore accentuée lorsque, par un nouveau tournant du texte, l'empiètement devient combat. On découvre alors que l'association des deux lieux ou des deux moments n'est pas une tranquille harmonie, que l'accouplement qu'elle forme n'est pas celui de l'amour, mais celui de la lutte: "le lieu lointain engendré autour de la sensation commune s'était accouplé un instant, comme un lutteur, au lieu actuel»(p. 874). Et Proust ajoute, superbement: «Toujours le lieu actuel avait été vainqueur ; toujours c'était le vaincu qui m'avait paru le plus beau ; si beau que j'étais resté en extase» (p. 875). Nous sommes là dans l'instant d'une "résurrection », et celle-ci n'est effective qu'au prix du déchirement du moi qui y assiste. Le passé ne revient et ne provoque l'extase du moi que quand cette réminiscence présente le double caractère $d u$ beau et $d u$ vaincu. Le souvenir est saisissant parce qu'il advient sans être parvenu à faire céder la défense que le lieu actuel oppose, «de toute la résistance de sa masse ", à son «immigration » (p. 874). Le moi se trouve ainsi traversé par un double désir, contradictoire: sa sensation veut "recréer autour d'elle le lieu ancien» (p. 874) et en même temps il veut que le lieu actuel résiste fermement à cette recréation, ne cède pas devant l'éventuelle intrusion de cette sensation de revenant.

Sinon, il n'y résisterait pas lui-même : "Et si le lieu actuel n'avait pas été aussitôt vainqueur, je crois que j'aurais perdu connaissance; car ces résurrections du passé, dans la seconde qu'elles durent, sont si totales qu'elles n'obligent pas seulement nos yeux à cesser de voir la chambre qui est près d'eux pour regarder la voie bordée d'arbres ou la marée montante; elles forcent nos narines à respirer l'air de lieux pourtant lointains, notre volonté à choisir entre les divers projets qu'ils nous proposent, notre personne tout entière à se croire entourée par eux, ou du moins à trébucher entre eux et les lieux présents, dans l'étourdissement d'une incertitude pareille à celle qu'on éprouve parfois devant une vision ineffable, au moment de s'endormir » (p. 875). La violence de la réminiscence fait chavirer le moi, le sidère, le livre à la plus grande inquiétude quant à lui-même, menace de le faire disparaître à luimême. Dans l'extase, il y a ce mouvement ébauché d'un absentement, d'une aliénation ; seule la victoire de l'actuel, du présent, de ce qui dans un premier temps devait être transpercé pour que le souvenir remonte, préserve le moi de cette perte de soi. L'expérience de la réminiscence est celle d'une déchirure: le moi y est ultimement sauvé par cela même qui d'abord s'opposait au souvenir, il lui faut simultanément aimer la beauté du vaincu et célébrer la force de résistance du vainqueur.

Les résurrections du passé sont "totales", mais seulement «dans la seconde qu'elles durent » (p. 875). La soudaineté, la fulgurance de l'événement, que désignait déjà le terme d'« éclair " plusieurs fois employé, sont ses caractéristiques essentielles. Elles s'opposent à la continuité de la mémoire volontaire, qui patiemment combine dans l'homogène, comme à l'endurance laborieuse dont a besoin l'intelligence pour parvenir à ses fins. Du coup, cela accorde aussi à la dimension de la «totalité » une tout autre signification : celle-ci n'est pas quantitative mais, si l'on peut dire, intensive. Le passé n'est pas restauré dans l'intégralité de ses déterminations constituées, il est rendu d'un trait, dans l'absolu de sa présence essentielle. La renaissance proustienne est plus 
proche du fragment des premiers Romantiques que des longues reconstitutions des artistes et des penseurs historicistes. Elle a les propriétés du fugitif.

Mais comment faire œuvre de ce fugitif? Comment «fixer " la "contemplation de l'essence des choses » (p. 876) ? Comment enchâsser un éclair dans les limites spatiales d'un livre et comment recueillir une cristallisation instantanée dans le temps long de l'écriture?

31 Toujours enfermé dans la bibliothèque-salon de l'hôtel de Guermantes, Marcel se dit que capturer des éclairs serait une entreprise décidément désespérée, si les sensations que leur survenue provoque en lui n'étaient pas comparables à une autre expérience, un peu plus tenace, à laquelle il se livre parfois par ailleurs. Il rapproche alors la réception des éclairs et cette autre pratique qui a lieu lorsque, assailli par une intuition vague, il tente de lui donner une signification distincte et s'efforce de la formuler de façon manifeste et bien délimitée. Le style pourrait presque évoquer les méditations cartésiennes :

Cependant, je m'avisais au bout d'un moment, après avoir pensé à ces résurrections de la mémoire, que, d'une autre façon, des impressions obscures avaient quelquefois [...] sollicité ma pensée, à la façon de ces réminiscences, mais qui cachaient non une sensation d'autrefois mais une vérité nouvelle, une image précieuse que je cherchais à découvrir par des efforts du même genre que ceux qu'on fait pour se rappeler quelque chose. (p. 878)

Or de quoi est-elle faite, cette autre expérience dont l'issue est une vérité nouvelle et une image distincte ? Elle consiste en un essai de " penser, c'est-à-dire de faire sortir de la pénombre ce que j'avais senti» (p. 879). L'exercice est celui d'un sondage et d'une remontée, d'une extraction de ce qui se trouve déjà dans le moi, mais enfoui profondément : deviné parfois pas les sens, mais non explicité par l'entendement. Tout se passe, ajoute le texte, «comme si nos plus belles idées étaient comme des airs de musique qui nous reviendraient sans que nous les eussions jamais entendus, et que nous nous efforcerions d'écouter, de transcrire » (p. 878) - et tout est dans cet oxymore d'un retour de ce qui n'a jamais été présent, d'un «à nouveau » de ce qui n'a pas eu de première occurrence. Ce qui nous revient est alors vraiment présent, mais d'une présence marquée de ce défaut initial, de cette absence originaire qui ne minimise en rien l'intensité de la sensation, qui n'empêche en rien l'écoute et la transcription, qui au contraire aiguise encore la première, lui donne une qualité singulière que l'écoute tente d'approcher et qui rend urgente et nécessaire la transcription.

Avec ce dernier terme, le texte aborde vraiment la poétique, le faire, la création. Mais il l'aborde en rapportant le passage du non-être vers l'être, la capacité de faire sortir de la pénombre, non pas au pouvoir d'un moi doté d'une aptitude affirmative, mais à l'antécédence d'une absence à soi. L'extraction et la transcription sont possibles parce que la sensation éprouvée ne renvoie d'abord à rien qui soit connu, maîtrisé, à rien que le moi ait déjà établi, à rien qu'il puisse maintenant reconnaître. Au contraire, l'effort que nous faisons d'écouter est lié au fait que nous ignorons ce qui dans l'écoute pourrait advenir. La réminiscence dont il s'agit avec ce que Proust appelle « résurrection» est le contraire d'une remémoration, en cela précisément que celle-ci tente de retrouver un objet identifié là où la première est engagée dans l'expérience d'assister au retour de ce qui n'a jamais eu lieu.

Ou plutôt, de ce à quoi le moi n'a jamais donné de lieu. Car le lieu, de fait, existe bien : dans la profondeur, dans l'obscurité intime du moi. Mais ce qui s'y trouve n'a pas été 
déposé par le moi lui-même : le lieu a été empli sans qu'il en décide, à son insu. Ces réminiscences et ces vérités «composaient un grimoire compliqué et fleuri » dont le «premier caractère était que je n'étais pas libre de les choisir, qu'elles m'étaient données telles qu'elles. Et je sentais que ce devait être la griffe de leur authenticité " (p. 879). C'est « la réalité » qui a « dicté » (p. 880) ce qui se trouve en ce lieu, c'est « la vie » qui l'a « communiqué » (p. 878), cela a été « donné »:

Je n'avais pas été chercher les deux pavés inégaux de la cour où j'avais buté. Mais justement la façon fortuite, inévitable, dont la sensation avait été rencontrée, contrôlait la vérité du passé qu'elle ressuscitait, des images qu'elle déclenchait, puisque nous sentons son effort pour remonter vers la lumière, que nous sentons la joie du réel retrouvé. Elle est le contrôle aussi de la vérité de tout le tableau, fait d'impressions contemporaines qu'elle ramène à sa suite avec cette infaillible proportion de lumière et d'ombre, de relief et d'omission, de souvenir et d'oubli que la mémoire et l'observation consciente ignoreront toujours. (p. 879)

L'« acte de création » est une lecture (p. 879), telle est la grande découverte que fait Marcel se remettant dans la bibliothèque de l'hôtel des émotions de l'incident précédent. Créer, écrire, faire une œuvre d'art, c'est entreprendre la lecture de ce "quelque chose de tout autre que je devais tâcher de découvrir » sous les signes et les images, sous le nuage, le triangle, le clocher, la fleur ou le caillou sur lesquels nous fixons notre attention; c'est s'efforcer de déchiffrer « une pensée qu'ils traduisaient à la façon de ces caractères hiéroglyphiques qu'on croirait représenter seulement des objets matériels" (p. 878). L'acte de création est une lecture, il consiste à relever, transcrire et interpréter les caractères hiéroglyphiques qui ont été tracés en nous sans nous, qui composent en nous à notre insu un livre secret, intime.

Quant au livre intérieur de signes inconnus (de signes en relief, semblait-il, que mon attention, explorant mon inconscient, allait chercher, heurtait, contournait, comme un plongeur qui sonde), pour la lecture desquels personne ne pouvait m'aider d'aucune règle, cette lecture consistait en un acte de création où nul ne peut nous suppléer ni même collaborer avec nous. (p. 879)

La vie a ainsi imprimé en nous des traces, et ce terme, employé par Proust, suggère bien le condensé de présence et d'absence que le moi est invité à explorer chaque fois que le livre de signes inconnus consent pour un instant à se rouvrir. Il a alors rendez-vous, certes, avec lui-même, avec l'empiètement de son présent et de son passé, de ce qu'il est et de ce qu'il a senti. Mais il est aussi confronté à cette étrange figure, spectrale, d'un moi marqué par son défaut, plongeant en lui dans des abysses où précisément il ne se trouve pas, où en tout cas il ne retrouve pas l'intégrité de ce que d'ordinaire il veut ou croit être. «Ne vient de nous-même que ce que nous tirons de l'obscurité qui est en nous ", se dit Marcel enfin déterminé à écrire, car il vient de comprendre que «le livre aux caractères figurés, non tracés par nous, est notre seul livre » (p. 880).

Le moi, pour faire œuvre, doit aller puiser là où il n'est pas, n'est plus ou n'est pas encore. Proust en tire la conclusion que «nous ne sommes nullement libres devant l'œuvre d'art, que nous ne la faisons pas à notre gré, mais que, préexistant à nous, nous devons, à la fois parce qu'elle est nécessaire et cachée, et comme nous le ferions pour une loi de la nature, la découvrir ». Et il ajoute que cette découverte de ce qui en nous nous préexiste est certes le fait de l'art, mais que l'art alors nous y offre, chaque fois, ce qui « nous reste d'habitude à jamais inconnu, notre vraie vie, la réalité telle que nous l'avons sentie et qui diffère tellement de ce que nous croyons, que nous sommes emplis d'un tel bonheur quand un hasard nous apporte le souvenir véritable » (p. 881). 
suggère l'affinité de la poiésis et de la joie - joie du miracle, de la merveille, de l'éclair soudain, de la résurrection. Mais un peu plus loin, c'est à la souffrance que, pour les mêmes raisons, la poiésis sera associée. Car la souffrance, tout autant que la joie, témoigne de l'état de non-liberté dans lequel, malgré nous, peuvent refaire surface les vérités hiéroglyphiques cachées sous les signes du monde et imprimées en nous.

Si peu que notre vie doive durer, ce n'est que pendant que nous souffrons que nos pensées, en quelque sorte agitées de mouvements perpétuels et changeants, font monter comme dans une tempête, à un niveau d'où nous pouvons la voir, toute cette immensité réglée par des lois, sur laquelle, postés à une fenêtre mal placée, nous n'avons pas vue, car le calme du bonheur la laisse unie et à un niveau trop bas.

Une objection traverse alors l'esprit de Marcel: «peut-être seulement pour quelques grands génies ce mouvement existe-t-il constamment sans qu'il y ait besoin pour eux des agitations de la douleur ».

Il l'écarte aussitôt : " encore n'est-il pas certain, quand nous contemplons l'ample et régulier développement de leurs œuvres joyeuses, que nous ne soyons trop portés à supposer d'après la joie de l'œuvre celle de la vie, qui a peut-être été au contraire constamment douloureuse » (p. 897).

\section{Berlin, Copenhague, La Flèche, Neubourg, Stockholm, Clermont-Ferrand, Port-Royal des Champs, Tübingen}

41 "Ce qui fait un événement de la rencontre d'un mot, d'une odeur, d'un lieu, d'un livre, d'un visage, n'est pas sa nouveauté comparée à d'autres "événements". C'est qu'il a valeur d'initiation en lui-même. On ne le sait que plus tard ».

Ce n'est pas à Proust que pensait Jean-François Lyotard quand il écrivait ces mots, mais à Walter Benjamin, aux "petites proses" de Sens unique et d'Enfance berlinoise "que Theodor Adorno aurait nommées des "micrologies" ». Ces proses, écrit Lyotard, «ne décrivent pas des événements de l'enfance, elles saisissent l'enfance de l'événement, elles inscrivent son insaisissable ${ }^{11} »$.

43 La qualité d'événement n'est pas due à la position qu'un fait occuperait par rapport à d'autres faits dans une série continue, mais à sa caractéristique intrinsèque d'être commençant, initial. En insistant sur l'émergence, le surgissement et l'advenue, il se pourrait que Lyotard fasse de Walter Benjamin, lecteur, traducteur, commentateur de La Recherche, un proche voisin de Proust. Ne retrouvons-nous pas ici l'indication d'une disjonction temporelle de la réminiscence («on ne le sait que plus tard»: l'instantanéité de l'événement et la conscience qu'on en a ne sont pas contemporaines, l'événement n'est pas davantage précédé par une connaissance qu'il viendrait confirmer ou infirmer), et ne constate-t-on pas là encore que l'événement échappe au pouvoir du sujet, qui le reçoit comme un « insaisissable»?

Mais la tonalité du texte de Lyotard semble trancher dans ce qui, chez Proust, se partageait entre joie et souffrance. L'événement, lit-on, «a ouvert une plaie dans la sensibilité. On le sait parce qu'elle s'est rouverte depuis et se rouvrira, scandant une temporalité secrète, peut-être inaperçue. Cette plaie a fait entrer dans un monde inconnu, mais sans jamais le faire connaître. L'initiation n'initie à rien, elle commence ». 
Une plaie est une blessure, un ébrèchement douloureux, une souffrance. La sensibilité s'en trouve altérée, son intégrité est mise à mal. Mais on comprend que cela ne va pas sans un certain avantage: l'entrée dans un monde inconnu. L'ouverture n'est pas seulement atteinte et lésion, elle est aussi gain et découverte. L'opacité s'écarte pour qu'advienne une autre chose : un monde. Ce monde demeure inconnu, irréductible à l'emprise, à la saisie. On n'en fait pas le tour, le fantasme de la totalité et celui d'un savoir exhaustif s'en trouvent déçus. Mais cela est une chance. L'événement n'est pas un moyen, une médiation, une étape dans la voie de la connaissance et de la conscience, il n'assume pas la fonction d'introduire à une figure ou à une signification supérieure, il ne conduit nulle part. Son sens est dans l'ouverture même.

La relation que l'on entretient avec cette ouverture est toujours une modalité du futur antérieur: l'événement aura été, la plaie aura ouvert ${ }^{12}$. On ne l'évoque que rétrospectivement, depuis ce qui s'est passé ultérieurement: depuis la réouverture. Celle-ci renvoie à l'événement comme au premier commencement, mais ce renvoi n'a pas les propriétés de la temporalité ordinaire, explicite, homogène. Entre la réouverture et l'ouverture, il y a un lien, mais pas celui qu'on se représente lorsqu'on dispose les faits comme s'ils étaient des points sur une ligne continue qui serait le temps. Ce qui ressurgit, c'est plutôt le fait même du commencement, chaque fois radicalement nouveau et pourtant rappelé dans la répétition. L'enfance berlinoise n'est pas tout ce que Walter a vécu trente ans avant de la relater, elle est ce qu'il est en train de vivre quand il écrit. Il ne peut écrire que parce que sa sensibilité est maintenant déchirée, et c'est cette déchirure actuelle qui mystérieusement communique avec les déchirures qui composent une enfance.

Quelques années plus tard, dans un entretien qui est alors en train de tourner autour de la question de la peinture, particulièrement celle de Barnett Newman, de Rothko et de Sam Francis, Élisabeth Weber rappelle à Jean-François Lyotard le passage cité du livre sur le postmoderne. Elle lui demande si «l'interrogation philosophique prend également naissance dans une telle plaie de la sensibilité ».

Réponse de Jean-François Lyotard :

Quant aux philosophes, il est certain que la philosophie ne commencerait même pas sans la plaie. Kierkegaard parle de son épine dans la chair. Le cas parle en faveur de mon idée, direz-vous. Mais il y a des plaies chez Descartes : les rêves sont des plaies, et le grand Trompeur. La plaie chez Pascal, elle saute aux yeux. Et même chez Hegel, la plaie s'exhibe dans son texte de jeunesse sur le scepticisme, un texte de douleur, où il est dit qu'on ne peut pas philosopher si l'on n'est pas passé par l'épreuve du nihilisme. Douleur que Hegel partage avec Hölderlin et Schelling. Chez les philosophes aussi, la blessure existe, une secrète douleur qui fait leur grandeur, leur acharnement. Mais aussi, dans la mesure où ils sont philosophes selon la tradition de la consolatio, ils essaient de cicatriser. Hegel dit que les plaies de l'esprit se cicatrisent toujours. Il est essentiel à l'esprit de se consoler. Cela s'appelle chez Hegel dialectique, usage de la sophistique des paradoxes à des fins rationnelles, etc. Mais la plaie dont nous parlons, de l'âme plutôt que de l'esprit, saigne sans cesse et demande, certes, à être soignée, mais aussi, à ne pas être soignée, à être respectée comme l'aleph. C'est le paradoxe, l'oxymoron de l'écriture même ${ }^{13}$.

Dans cette réponse, la " plaie » reçoit une détermination apparemment moins ambiguë encore que dans la citation précédente. Ici, elle est exclusivement placée du côté de la douleur, de la blessure, du saignement.

L'ambivalence demeure pourtant, elle est même portée à son paroxysme, quand Lyotard indique ce qui, selon lui, constitue la singularité de la poiétique philosophique. 
Celle-ci est expérience de la plaie, connaissance de la douleur, acquiescement à la souffrance, probité d'un affrontement de la blessure qui ne la dissimule pas, ne la déguise pas, ne l'idéalise pas avec trop d'empressement; et elle est aussi expérience de la cicatrisation, exercice de consolation, transfert de l'insaisissable dans une rationalité lénifiante.

51 La philosophie soigne l'âme et en même temps répond à l'injonction que l'âme lui adresse de ne pas être soignée.

Car il y a dans l'âme quelque chose qu'il ne faut pas vouloir soigner, au risque d'anéantir, de faire taire irrémédiablement l'injonction, l'appel, l'envoi qui la motive : précisément ce qui en elle est événement, ouverture de la plaie, commencement, ce que Lyotard désigne maintenant par la première lettre hébraïque: l'aleph. Incision douloureuse, sans laquelle rien ne se passerait, ébrèchement, lacération qui creuse l'endroit d'un advenir.

La philosophie est écriture, au sens où elle s'exerce dans le lieu impossible d'un paradoxe, d'un oxymoron. Elle ne serait pas elle-même si elle déniait totalement la plaie, si elle laissait croire à un commencement dans la plénitude, dans la certitude, dans le dogme ; elle ne serait pas elle-même si elle renonçait totalement à la consolation, au soin, à la cicatrisation, si elle se faisait exclusivement plainte, ou prière, ou cri. Elle est dans le geste contradictoire, incohérent, pathétique d'accueillir la plaie comme ce qui l'engage et la met en mouvement, mais d'esquisser en même temps le protocole de sa guérison, mais d'interrompre rapidement ce protocole pour que l'événement demeure possible, pour que puissent encore avoir lieu des commencements, des réouvertures de la plaie qui engagent, envoient, appellent, ordonnent, mettent en mouvement la pensée.

\section{Manchester, Colmar, Kissingen}

Le peintre Max Ferber que le narrateur rencontre dans son atelier dans la Manchester «noire de suie et en complète décrépitude» de 1966 manifeste une étrange prédilection pour la poussière. Elle lui est, lui dit-il, « plus familière que la lumière, que l'air, que l'eau ». Sebald constate, de fait, que nulle part Ferber « ne se trouvait plus à l'aise que là où les choses ont le droit de rester où elles sont, sans qu'on les dérange, adoucies par la scorie noire et veloutée qui se dépose quand la matière, par touches imperceptibles, se décompose pour retourner au néant ${ }^{14} »$.

Cette affinité avec la poussière est directement liée à la pratique artistique de Ferber. Celui-ci ne cesse en effet de gratter sur la toile les couleurs qu'il y a appliquées en grandes quantités (p. 210) et de biffer énergiquement, à l'aide d'« un chiffon de laine saturé de charbon », les nombreux traits de fusain qu'il trace sur le papier, de sorte que «ce crayonnage [...] n'était en réalité rien d'autre qu'une production de poussière » (p. 211-212). Le narrateur finit même par s'étonner qu'au terme d'une journée de travail, le peintre, "à partir des rares lignes et ombres ayant échappé à l'anéantissement, [ait] composé un portrait d'une grande spontanéité ». Il sait pourtant que dès le lendemain ce portrait sera «infailliblement effacé » par Ferber, "pour lui permettre à nouveau, sur le fond déjà fort compromis par les destructions successives, d'exhumer, selon son expression, les traits du visage et les yeux en définitive insaisissables de la personne » (p. 212). Et Sebald ajoute : «Quand il se décidait enfin [...] à se dessaisir d'un tableau [...], on croyait avoir devant les yeux un portrait issu d'une 
longue lignée d'ancêtres aux visages gris, surgis de leurs cendres pour continuer à hanter sans fin le support malmené » (p. 212).

Ferber est-il un poiète, son art est-il «cause du passage du non-être vers l'être »? Assurément, puisqu'il produit des œuvres, qui bénéficieront même, nous l'apprendrons plus tard dans le texte, d'une reconnaissance et d'un certain succès. Mais il l'est d'une manière complexe: le mode de fabrication utilisé accorde au moins autant d'importance à la destruction qu'à la construction de formes, les formes survivantes relèvent au moins autant de la visitation spectrale que de la présence assurée. L'œuvre, mais aussi la vie de Max Ferber, témoignent incessamment d'une lutte dans laquelle toute consistance affirmée se trouve immédiatement menacée par le mouvement contraire de l'anéantissement, de la disparition. La poussière, la cendre (qu'on retrouve, à la fin du texte, dans le teint du visage de Ferber mourant), sont des marques tangibles de cette lutte, comme le sont les reflets métalliques produits par la poussière sur les mains du peintre, qui évoquent soudain le corps d'un laborantin devenu luimême, après des années d'absorption d'argent, une sorte de plaque photographique ${ }^{15}$.

Vers la fin du texte, l'écrivain parle de l'extrême difficulté qu'il a ressentie à l'écrire et même des «scrupules » dont il s'est trouvé assailli pendant sa rédaction, scrupules qu'il attribue «autant à l'objet de mon récit auquel je croyais, quoi que je fisse, ne pas rendre correctement justice, qu'à une mise en question de la possibilité de toute écriture» (p. 300). Aurait-il soupçonné quelque chose de discutable, voire de répréhensible, dans le fait de tirer un parti unilatéralement positif du récit d'une pratique et d'une existence dans lesquelles le mouvement de la disparition était au moins aussi manifeste et signifiant que celui de la création? Quoi qu'il en soit, c'est comme si l'antagonisme qui déchire de l'intérieur la poétique de Ferber avait ôté toute évidence à la tentative de la relater : dans les deux cas, la lutte avec l'impossible est devenue la condition inapaisable de la fabrication.

Pour parvenir à l'œuvre, il faut chaque fois, pour chaque tableau, pour chaque page, découvrir la "porte dérobée» (p.232) qui, dénouant un peu le milieu saturé dans lequel se trouve d'abord confiné le peintre ou l'écrivain, ouvre sur l'espace du figurable ou du racontable; mais le chemin alors entrevu finit inexorablement par se perdre ou s'interrompre.

Le texte accorde, comme toujours chez Sebald, une importance singulière aux voyages. Ils sont ici multiples: l'écrivain raconte dans les toutes premières pages son propre trajet d'Allemagne à Manchester en 1966, et plus loin on lit le récit du même itinéraire suivi dans de tout autres conditions, en $1939^{16}$, par Ferber. S'ajoutent plusieurs déplacements en sens inverse, de Manchester vers d'autres destinations, voyages initiatiques au bout desquels est révélé à chacun des protagonistes, à Ferber puis au narrateur parti sur les traces du premier, une partie de ce qui constitue la vérité de son art et de sa vie.

60 La construction de l'ensemble suggère ainsi une forme de symétrie entre le choc ressenti par Ferber à Colmar devant le retable d'Issenheim et celui qu'éprouve le narrateur lors de sa visite du cimetière juif de Kissingen. Ferber ressent la peinture de Grünewald, qui «tord tous les membres sans exception, pigmente les couleurs comme une maladie ", comme "profondément conforme à [sa] personnalité ». La souffrance, "émanant des personnages représentés, recouvre la nature entière pour ensuite refluer des paysages éteints et pénétrer les figurations humaines de la mort »; elle est comme une "monstruosité [...] en mouvement» qui monte et descend en lui 
« exactement comme les oscillations d'une marée ». Cette expérience lui suggère une méditation sur la douleur qui "parvenue à un certain stade [...] abolit les circonstances de son apparition, la conscience, et partant s'abolit elle-même", tandis que «la souffrance morale, elle, est pratiquement infinie » (p. 222-223).

De nombreuses années plus tard, le narrateur, visitant le cimetière juif de la petite ville qui fut le berceau de la famille de Ferber, se sent soudain à son tour personnellement interpellé, comme si les sépultures, faisant en lui événement, ouvraient une plaie dans sa propre chair.

Un frisson me parcourut devant une tombe où reposait Meier Stern, décédé le 18 mai, soit le jour de ma naissance, et de même le symbole de la plume d'oie sur la stèle de Friederike Halbleib, morte le 28 mars 1912, provoqua en moi un trouble dont je dus m'avouer que je ne parviendrais jamais à percer complètement les raisons. Je me l'imaginais écrivain, penché solitaire et le souffle court sur son travail, et à présent que j'écris ces lignes, il me semble que c'est moi qui l'ai perdue et que la douleur de sa perte reste entière malgré le long temps écoulé depuis sa disparition (p. 291-292).

L'événement de cette perte interrompt chez Sebald le trajet entrepris après la lecture des notes rédigées par Luisa Lanzberg, la mère de Max Ferber. L'écrivain avait tenté de s'approprier le temps évoqué, subjectif et historique, qui d'une enfance et d'une jeunesse juives plutôt sereines, conduisit aux traumatismes de la première guerre mondiale et de l'écrasement de la révolution de Munich. Mais le chemin de la mémoire, compris comme une sorte de reconstitution désirée, échoue sur une cristallisation qui le rend vain, illusoire, infertile.

Chez Ferber, c'est apparemment l'inverse. L'émotion provoquée par la peinture de Grünewald semble d'abord rouvrir les voies du passé. «À Colmar en tout cas [...] j'ai commencé à me souvenir» (p.224), confie Ferber au narrateur, avant d'évoquer longuement ses relations avec son père et de façon générale la situation de sa famille dans l'Allemagne nazie. Mais au terme de ces remémorations nombreuses et précises, il $\mathrm{y}$ a des blancs, des trous, et la conclusion finalement tirée par Ferber que « le temps [...] n'indique rien d'autre que les fluctuations de l'âme. Il n'existe ni passé ni avenir. En tout cas pour moi » (p.236). Il y a, par exemple, cette «tache d'amnésie » dont « la raison et l'ampleur [...] étaient restées une énigme » (p. 227). Il y a l'échec, après toute une année d'effort, de peindre un portrait qui serait la représentation d'un souvenir précis, mais que l'artiste finit par brûler après avoir constaté « qu'à son avis il n'existait aucun point de repère, fût-il approximatif, pour rendre compte de l'étrangeté de la vision à la base de son tableau » (p. 228). Il y aussi les images irréelles, spectrales, qui lui viennent quand il pense à l'Allemagne et à la langue allemande, telle cette «dame élégante, en robe de bal taillée dans de la soie grise de parachute ", qui fait irruption dans son atelier : "Elle enlève son chapeau, ses cheveux ruissellent sur ses épaules, elle ôte ses gants d'escrimeuse, les jette ici, sur le guéridon, et se penche sur moi. Je ferme les yeux et perds connaissance. La suite, je ne la connais pas » (p. 237). Ferber ajoute : «Je crois que la dame grise ne comprend que sa langue maternelle, l'allemand, que depuis 1939, depuis les adieux à mes parents sur l'aéroport munichois d'Oberwiesenfeld, je n'ai plus jamais parlé une seule fois [...] Il est possible, continua Ferber, que cette perte et cet ensevelissement de la langue aient quelque chose à voir avec le fait que mes souvenirs ne remontent pas plus loin qu'à ma neuvième ou dixième année ». Les indications données par ailleurs dans le texte permettent de dater précisément cette syncope de la mémoire : 1933-1934. 
64 illustrés » qu'il a intitulé Les émigrants, W. G. Sebald tente-t-il d'approcher une vérité de la poiésis ? La manière singulière de l'artiste, immergée dans la terrible histoire du $\mathrm{xx}^{\mathrm{e}}$ siècle européen et partiellement dans son intenable dénégation, lui parle-t-elle de ce que sont désormais devenus toute fabrication, tout " passage du non-être vers l'être ", y compris la fabrication de textes à laquelle se livre Sebald lui-même ? Parmi bien d'autres, deux moments du récit illustreraient alors, sans rien prétendre démontrer, quelque chose de cette vérité. Dans l'un, le narrateur revoit Ferber dans la cantine sordide où ils avaient l'habitude de se retrouver, bien que l'on y servît une « tambouille effroyable, mi- anglaise, mi- africaine ", au cœur du quartier portuaire en plein délaissement. Il est assis «devant une fresque peinte par une main anonyme et représentant une caravane surgie des profondeurs du tableau pour franchir la houle des dunes et se diriger droit sur le spectateur ». L'association est immédiate : « Quand Ferber avait travaillé au fusain et que la fine poudre de poussière donnait à sa peau des reflets métalliques, il me semblait qu'il venait tout juste de sortir du désert ou que rentrant dans le tableau, il allait incessamment y retourner » (p. 214-215). compagnie de la reine Victoria, « dans un cabinet fort poussiéreux, où visiblement plus personne n'avait remis les pieds depuis des années [...] et dans lequel je reconnaissais, après quelque hésitation, la salle de séjour de mes parents ». Un inconnu est assis là, tenant sur ses genoux «une maquette du temple de Salomon peinte en dorure et confectionnée en bois de sapin et papier mâché ». Il se présente à Ferber et lui explique qu'il a mis sept ans à " réaliser de ses mains cette reproduction fidèle aux indications fournies par la Bible » et qu'il va maintenant « de ghetto en ghetto pour la présenter ». Il l'invite à constater qu'« on reconnaît le moindre créneau, le moindre rideau, le moindre seuil de porte, le moindre objet de culte ». L'épisode se termine par ces mots : «Et moi, dit Ferber, je me penchais sur ce petit temple et je savais pour la première fois de ma vie à quoi ressemble une véritable œuvre d'art » (p. 229-230).

À quoi ressemble-t-elle ? Peut-être Ferber dans son délire pénétrant y discerne-t-il une invitation paradoxale à reconnaître ce que l'on n'a jamais vu, ce qui a inexorablement disparu et n'est plus attesté que dans des traces, ici d'écriture, auxquelles il paraît pathétiquement dérisoire de vouloir redonner un statut de signes pleins. Ces traces, éphémères et magnifiques, seraient exhumées du désert, et elles y reconduiraient inéluctablement. Elles écarteraient un peu le sable, la cendre et la poussière pour apparaître, et s'en laisseraient bientôt recouvrir à nouveau, car on ne saurait creuser les choses jusqu'à atteindre le socle où elles reposeraient, suaves et pures, préservées des altérations du monde et du temps

\section{Pays de Ous, entre Arabie et Idumée}

67 «Je ressemble à la poussière et à la cendre!"; «je m'abîme et me repens, / sur la poussière et sur la cendre! ». Les occurrences sont nombreuses dans Le livre de Job, une quinzaine. La poussière est parfois signe de compassion, comme celle que les amis de Job répandent sur leur tête au début du texte. Elle indique l'humilité ( j’ai enfoncé ma corne dans la poussière »), la souffrance de l'opprobre («Maintenant que dans la poussière je me couche »), la matière dont est fait l'homme (« Tu m'as fait avec de l'argile et en poussière tu me feras retourner»), la mort qui l'attend («nous 
enfoncerons-nous ensemble dans la poussière? ", " ensemble dans la poussière ils se couchent / et les vers les recouvrent $! »)^{17}$. Alliée à la cendre, elle nomme un état qui apparait d'abord comme un accident ou une aberration, mais que le rappel de ce qui constitue le propre de l'humain inclura dans sa condition et son espace spécifiques.

Le malheur frappe brusquement un homme prospère, pieux et juste: troupeaux décimés et meurtre des serviteurs, destruction de la maison et mort de tous ses enfants, maladie ravageuse (lèpre ?). Le texte est poétique, il n'est ni un récit, ni un exposé doctrinaire, plutôt une longue méditation sur la douleur, méditation partagée, plainte et discussion alternées, parole d'interrogation et parole polémique, allégations et heurts d'arguments. Les amis de Job, venus le seconder et le consoler, expliquent le mal comme une sanction, développent une logique de la compensation, de la nécessaire balance entre châtiment et faute. Job récuse cette logique, faisant de Dieu, au risque du blasphème, le seul responsable, arbitraire, sans raison, de ses souffrances. Au terme de ces controverses, Iahvé prend la parole. Il accuse Job d'avoir outrepassé son rang en s'érigeant en juge de la toute-puissance divine et finalement c'est en sa faveur à lui, Job, qu'il tranche. C'est lui qui a proféré des vérités et non ses amis, qu'il condamne à offrir un holocauste.

Pour eux, le traumatisme, qui doit forcément être expliqué, ne peut avoir pour signification ultime qu'une déviance à corriger. Le mal est la rupture d'un équilibre, d'un ordre économique qu'il s'agit de rétablir, comme on remet à niveau égal les deux plateaux d'une balance. C'est une aberration, sa cause est dans un écart commis qui contredit la relation normale des hommes avec Dieu. Le rappel des certitudes dogmatiques et l'observance de la forme la plus stricte de cette normalité qui fait loi peuvent seuls rétablir ce qui s'est trouvé dérangé.

70 Ce que Job oppose à cela est beaucoup plus risqué et difficile. C'est comme si pour lui le trauma, dont il souffre, dont il se plaint, qu'il dénonce, ne devait pourtant pas être totalement compensé, annulé ou dénié au profit d'un retour à l'équilibre. Comme s'il avait ouvert un vide, une béance, et que ce vide et cette béance ne devaient pas être complètement recouverts, parce que se trouve en eux un sens éminent, supérieur à tout enseignement dogmatique, à toute assurance institutionnelle.

71 Job le répète inlassablement : il se situe hors de tout dispositif ou de tout système de la consolation comprise comme colmatage de la brèche et rétablissement de la norme. L'épreuve qui est la sienne l'engage au-delà de toute relation de type contractuel (XVII, 3-4). Car le mal qui le frappe le transporte sans intercession face à la puissance divine, qui n'est soumise elle-même à aucune règle économique de régulation, de distribution équilibrée et de rachat équivalent, à aucune logique de la rétribution (IX, X). Dieu luimême est incommensurable, ses décisions ne sont pas explicables, son arbitraire n'est réductible à aucune consécution de cause et d'effet, à aucun schéma qui le rendrait compréhensible mais ne serait au fond que projection anthropomorphique.

La leçon qu'en tire Job est simultanément désespérée et libératrice. Elle porte sur la parole, sur sa propre parole (cf. IX, 20-21 et 27-34). Quoi qu'il dise, cela est vain. S'il est condamné par l'arbitraire divin, aucune plainte, aucun argument, aucun plaidoyer ne viendra réparer quoi que ce soit. La relation qui s'est instaurée n'est pas de l'ordre de l'échange, ni même du procès en justice : elle excède infiniment toute pesée et toute rémunération. Rien à faire, donc; mais c'est précisément cette impuissance qui ouvre l'espace d'une parole possible. C'est justement parce que Job ne se trouve pas dans une 
situation assimilable au juridique ou à l'économique qu'il parle sans crainte : « Puisqu'il n'en est pas ainsi, moi avec moi-même / je parlerai et ne le craindrai pas » (IX, 35).

Le Livre de Job est peut-être d'abord un poème sur la parole. Sur sa possibilité et son impossibilité, sur son effectivité et sa limite, sur les conditions de sa tenue et de son inscription. La parole de Job n'a pas de répondant : c'est sa souffrance, et c'est aussi son commencement, son événement. Au lieu de la réponse (à la plainte, à la question, à l'appel), le silence, le lieu déserté ${ }^{18}$, l'incommensurable au-delà de toute communication : «Je crie vers toi et tu ne me prêtes pas attention / je me tiens debout et tu ne me prêtes pas attention / tu es devenu cruel pour moi » (XXX, 20-21). Pourtant Dieu est bien destinataire de la parole de Job : « ma clameur est arrivée jusqu'à Eloah » (XVI, 19-20), «il me connaît, quand je marche et quand je m'arrête » (XXIII, 10); mais être destinataire ne l'oblige en rien, son décret est au-delà de toute obligation : «il a opté et qui l'en empêchera? » (XXIII, 13).

Cela condamne-t-il Job au mutisme ? S'il n'y a aucune alternative à la disparition, celle de Job qui, mort, sera soustrait à tout regard, y compris celui de Dieu (VII, 8), et qui maintenant, effrontément, demande à Dieu de s'éloigner pour que son agonie au moins soit supportable : «Retire-toi de moi...» (X, 21-22), que reste-t-il à dire ? Il reste peu, cela tient en deux vers, mais cet in extremis, dans la scansion qu'il contient d'une question, d'une affirmation et d'un vœu, désigne peut-être le lieu spécifique de la parole humaine. Job dit: "Qui me donnera quelqu'un qui m'écoute? / Voilà ma signature ! Que Shaddaï me réponde !» (XXXI, 35).

"Voici ma signature, précise Édouard Dhorme, en hébreu "mon taw", dernière lettre de l'alphabet, en forme de croix, qui servait de signature aux illettrés ${ }^{19}$ ». Par cette exclamation - « Voici ma signature ! » - Job accorde à sa propre parole de plainte et de requête la forme affirmative d'une attestation, d'un acte garantissant son existence et sa légitimité. Mais cette forme est aussi celle qu'utilisent les illettrés, ceux qui entretiennent avec la langue une relation marquée par une faiblesse ou déficience. Job pose sa parole comme effectivité, et en même temps il en désigne l'infirmité. Plus tôt (VI, 3) il avait dit : «mes paroles sont bégayées » - comme celles de Moïse ? Maintenant, il les confie à l'efficacité et à l'indigence d'un petit signe, dernière lettre qui, reliée à la désignation par Jean-François Lyotard de la plaie comme l'aleph, nous aura fait parcourir tout l'alphabet.

76 Et peut-être en effet l'épreuve du trauma fait-elle comprendre à Job ce qu'il en est de la parole humaine: affirmation de soi, mais loin de toute suffisance; attestation et interpellation, mais qui ne sauraient décider elles-mêmes de la présence d'un répondant. Ce que signe Job avec son taw, c'est l'attente d'un don: d'un événement, d'un aleph - «Qui me donnera quelqu'un qui m'écoute?». La parole n'est pas décision, elle est demande, attente. Il ne dépend pas du locuteur que sa parole soit écoutée, ni répondue. La réponse vient d'ailleurs, elle est trace d'une altérité incommensurable. «Que Shaddaï me réponde! » n'est pas un ordre, un impératif, c'est une prière, un vœu, un appel lancé dans le désert du monde.

77 La parole de l'homme est irréductible au plan du savoir, de la connaissance, comme elle est irréductible au plan du droit; elle est essentiellement réponse à un envoi antérieur et quand cet envoi est absent ou incompréhensible, quand il prend la forme de la plaie et du trauma, elle est adresse à l'autre au-delà de toute symétrie, de toute communauté de langage présumée, de toute équivalence exigée - « Puis appelle et, moi, je répondrai, 
/ ou bien je parlerai et tu me répliqueras. » (XIII, 22). En l'occurrence, elle est adresse à l'autre pour que lui soit donné l'événement d'une réponse.

78 Cet événement n'a pas lieu, c'est ce que dit Job désespérément : «Si je crie à la violence, je ne reçois point de réponse, / j'ai beau clamer : il n'y a point de jugement » (XIX, 7). Pourtant, en une occurrence, il dit le contraire, dans des termes qui paraissent d'abord difficilement compatibles avec l'ensemble du propos : « Un objet de risée pour son ami, je le suis, / moi qui appelle Eloah et à qui il répond. / Il est objet de risée, le juste parfait!» (XII, 4). Dans la mesure où cette seule occurrence dans le texte associe la réponse d'Eloah et la qualité de Job d'être un juste, elle permet peut-être de distinguer deux modalités de la parole humaine. La première appartiendrait à une logique de la réciprocité, de l'échange symétrique et de l'équilibre : parole de communication et de socialité. La seconde, elle, s'inscrirait dans un face-à-face déséquilibré et asymétrique, parole lancée par-dessus la béance, l'absence, le désert, la poussière et la cendre, sans assurance d'être entendue ni répondue, se risquant là où précisément fait défaut toute logique de compensation. Ce serait la parole du juste, à laquelle Eloah « répond » sous d'autres formes que l'échange explicite, que la présence vérifiée, que le comblement de la brèche qu'ouvraient le trauma, la question et la plainte, et parfois jusqu'au maintien de cette brèche, jusqu'à la désertion, le retrait, le silence.

79 Cette parole-là est celle dont Emmanuel Levinas posait qu'elle « se dit ne fût-ce que par le silence gardé » et qu'elle témoigne d'un " écart qui s'accuse inévitablement entre Autrui comme mon thème et Autrui comme mon interlocuteur, affranchi du thème qui semblait un instant le tenir ", écart qui " conteste aussitôt le sens que je prête à mon interlocuteur ${ }^{20} »$. Le Livre de Job construit patiemment cette distinction, entre un mode de langage qui prend Dieu comme "thème " et lui prête toutes sortes de significations, et un mode de langage qui consent à la contestation de toutes ces significations, posant à titre d'interlocuteur un autre situé au-delà de toute thématique et susceptible de répondre jusque dans le « silence gardé ».

Ainsi, la requête de Job, l'appel qu'il signe de son taw ne visent pas à faire entrer Dieu dans la relation symétrique de la communication - et c'est ce que n'arrivent pas à comprendre ses amis consolateurs - mais à invoquer sa présence selon un mode de relation tout autre. Ce mode correspondrait à ce que Levinas appelait «la profonde essence du langage », qui selon lui se caractérise par « l'irréversibilité de la relation »: "Celui qui me parle et qui, à travers les mots, se propose à moi conserve l'étrangeté foncière d'autrui qui me juge; nos relations ne sont jamais réversibles. Cette suprématie le pose en soi, en dehors de mon savoir ». Levinas ajoutait : «Le langage ne peut se parler, en effet, que si l'interlocuteur est le commencement de son discours, s'il reste, par conséquent, au-delà du système, s'il n'est pas sur le même plan que moi (...) Dès lors, la vérité, exercice souverain de la liberté, devient possible ${ }^{21}$ ». C'est ce que Job demande quand il s'écrie: «Que Shaddaï me réponde!»: qu'ait lieu, devant moi, l'événement d'une parole qui soit commencement (aleph). Alors seulement la réponse, qui n'est aujourd'hui qu'absence, silence et désert, sera vérité, parole traversant, sans les abolir, absence, silence et désert.

81 Ainsi, lorsque Job demande que l'interlocution ait lieu sous la forme d'un procès, d'une confrontation face-à-face (XXIII, 2-7), ceux-ci ne sont en rien réductibles aux procédures d'arbitrage qui interviennent dans les contentieux entre humains (IX, 32-34; XVI, 21). Le texte les décrit plutôt comme lutte, combat (XIX, 8-12 ; XXXVIII, 3 ; $\mathrm{XL}, 7)$, rien qui évoque l'égalité, la réversibilité, l'équilibre. 

Qui donnera que soient écrites mes paroles! / Qui donnera que sur l'airain elles soient gravées, / qu'avec un burin de fer et de plomb, / pour toujours sur le roc elles soient sculptées!» (XIX, 23-24). La condition de l'écriture, de l'œuvre, c'est, une nouvelle fois, le don. Ce n'est pas le sujet qui en décide, il faut que depuis sa distance un autre en soit le commencement, l'événement. La suite immédiate du poème nomme cet autre («mon défenseur »), dans une tonalité messianique : « le dernier sur la terre, il se lèvera » (XIX, 25-27). La poiésis n'est pas un acte de volonté, mais de vérité, parce qu'elle suppose un envoi ou une adresse qui se situe "au-delà du système", depuis une " étrangeté foncière ». Elle est toujours elle-même réponse, entente d'une parole qui la précède, qu'elle reçoit comme un don, à laquelle elle se mesure. L'œuvre humaine n'est pas originaire. Elle n'a lieu qu'en écho à un événement dont elle est destinatrice. Elle bégaye des réparties, des répliques, qui sont à leur tour des demandes d'interlocution et des questions lancées au désert.

Que l'homme ne soit pas poiète originaire, c'est ce que dit Iahvé quand enfin, « du sein de la tempête " (car l'événement ne peut avoir lieu que dans la disjonction, dans la violence de l'ébrèchement, dans la déchirure de la familiarité), il consent à répondre aux appels de Job. « Où étais-tu quand je fondai la terre ?» (XXXVIII, 4) - c'est là, au fond, la seule teneur de sa parole, dans les quatre chapitres (XVIII-XLI) de son discours. Il y a un poiète originaire, un Créateur, mais l'homme, par rapport à la poiésis du monde, vient toujours trop tard, toujours dans la perte de l'origine, toujours dans la vacance, toujours dans la répétition de l'événement et non dans l'événement même. L'homme est l'absent du commencement, du principe, de l'événement, de l'instauration de la loi, de la source des phénomènes naturels, de l'aleph. Il n'est ni l'architecte du monde, ni celui qui réalise sur terre ce qui est écrit dans les cieux (XXXVIII, 33) - parce que son temps à lui n'est pas contemporain de l'origine.

Iahvé remet Job à sa place : place d'un humain défini par le retard, la non-coïncidence, l'espacement. Et à cela, Job n'a rien à répondre d'autre que: «je m'abîme et je me repens, / sur la poussière et sur la cendre !». Cette réponse n'est pas un renoncement à l'existence ou à l'activité, au contraire. La parole à laquelle elle répond ne voue pas Job au mutisme ; elle n'empêche pas qu'il aura été « donné » à ses paroles d'être "écrites ", précisément dans ce Livre de Job que nous sommes en train de lire; elle n'interdit en rien à l'homme d'être poiète. Au contraire. L'homme est poiète quand il acquiesce à sa condition de mortel, quand il ne dissipe pas la poussière, quand il se tient dans l'espace d'une relation avec une altérité irréductible, relation faite autant de vacance que de présence. Dans cet espace, le trauma peut être autre chose qu'une aberration. Il peut aussi être événement de la sagesse, sagesse qui «a été cachée à l'homme » (XXVIII) et que celui-ci voisine quand, juste, il accepte de côtoyer ce qu'il ne maîtrise pas, ce dont il n'est pas l'origine. Le trauma a alors à voir avec la poiésis, quand celle-ci accepte que ses œuvres soient des réponses, dans une relation dissymétrique, non réversible, quand elle accepte de porter en elle la trace de la vacance qui, au lieu de l'origine, marque l'homme, lui est constitutive, le limite en le définissant comme être tardif et simultanément lui ouvre les espaces infinis de la parole bégayée et de la vérité, " exercice souverain de la liberté ». 


\section{BIBLIOGRAPHIE}

Aristote, Ethique à Nicomaque, traduction et notes de J. Tricot, Paris, Vrin, 1987.

Blanchot Maurice, L'Entretien infini, Paris, Gallimard, 1969.

Le livre de Job, traduction d'Édouard Dhorme, La Bible. Ancien Testament, Paris, Gallimard, Bibliothèque de la Pléiade, tome II, 1959.

Levinas Emmanuel, Totalité et Infini, La Haye, Martinus Nijhoff, 1974.

Lyotard Jean-François, Le Postmoderne expliqué aux enfants, Paris, Galilée, 1986.

Mallarmé Stéphane, CEuvres complètes, Paris, Gallimard, bibliothèque de La Pléiade, 1945.

Montaigne Michel de, Essais, Paris, Garnier-Flammarion, 1969.

Platon, Le Banquet, traduction de Luc Brisson, Paris, Garnier-Flammarion, 1998.

Plotin, Ennéades, traduction d'Émile Bréhier, Paris, Les Belles Lettres, 1931.

Proust Marcel, À la Recherche du Temps perdu. Le temps retrouvé, Paris, Gallimard, bibliothèque de la Pléiade, tome III, 1954.

Rousseau Jean-Jacques, Les rêveries du promeneur solitaire, Paris, Garnier-Flammarion, 2011.

Sebald Winfried Georg, Les émigrants, traduction de Patrick Charbonneau, Paris, Actes Sud, 1999, Gallimard, Folio, 2003.

Weber Élisabeth, Vidal-Naquet Pierre et al., Questions au judaïsme. Entretiens avec Elisabeth Weber, Paris, éditions Desclée de Brouwer, 1996.

\section{NOTES}

1. Platon, Le Banquet, 205 b-c, traduction de Luc Brisson, Paris, Garnier-Flammarion, 1998.

2. Ibid.

3. Plotin, Ennéades, V, 8, traduction Émile Bréhier, Paris, Les Belles Lettres, 1931.

4. Aristote, Ethique à Nicomaque, traduction et notes de J. Tricot, Paris, Vrin, 1987, p. 31.

5. Montaigne, Essais, Livre II, chapitre VI, « De l'exercitation », Paris, Garnier-Flammarion, 1969.

6. «Je ne peins pas l'être. Je peins le passage... » : Montaigne, Essais, Livre III, chapitre II, « Du repentir ", op. cit.

7. Jean-Jacques Rousseau, Les rêveries du promeneur solitaire, Deuxième promenade (1776), Paris, Garnier-Flammarion, 2011.

8. Stéphane Mallarmé, Avant-dire au Traité du verbe de René Ghil, 1886, repris in "Crise de vers ", Stéphane Mallarmé, Euvres complètes, Paris, Gallimard, bibliothèque de La Pléiade, 1945, p. 368.

9. La séquence en question se trouve in Marcel Proust, La Recherche du Temps perdu, Le temps retrouvé, Paris, Gallimard, bibliothèque de la Pléiade, 1954, tome III, p. 866-917. Dans les lignes qui suivent, à une exception près, nous nous en tenons aux p. 866-881.

10. Ibid., p. 872 («l'être qui était rené en moi»), p. 874, p. 875 («l'être par trois ou quatre fois ressuscité en moi »), p. 878.

11. Jean-François Lyotard, Le Postmoderne expliqué aux enfants, Paris, Galilée, 1986, p. 141-142.

12. Le futur antérieur est pour Lyotard, dans le même livre, le mode d'être de ce qu'il appelle le postmoderne. Ibid., p. 33 : «L'artiste et l'écrivain travaillent donc sans règles, et pour établir les règles de ce qui aura été fait. De là que l'œuvre et le texte aient les propriétés de l'événement, de 
là aussi qu'ils arrivent toujours trop tard pour leur auteur ou, ce qui revient au même, que leur mise en œuvre commence toujours trop tôt. Postmoderne serait à comprendre selon le paradoxe du futur (post) antérieur (modo). »

13. Jean-François Lyotard, in Questions au judaïsme. Entretiens avec Élisabeth Weber, E. Weber, P. Vidal-Naquet et al., Paris, Desclée de Brouwer, 1996, p. 204-205.

14. Winfried Georg Sebald, "Max Ferber», in Les émigrants, traduction Patrick Charbonneau, Paris, [Actes Sud, 1999], Gallimard, Folio, 2003, p. 211.

15. Ibid., p. 215-216. Le phénomène décrit est en quelque sorte l'inverse du suaire de Turin : non pas le corps imprimant son image sur un linge l'entourant, mais le corps se faisant lui-même surface d'inscription.

16. Ibid., p. 244-246, récit qui contredit une première version racontée par Ferber le faisant arriver à Manchester en 1943, ibid., p. 218.

17. Le livre de Job, respectivement chapitres XXX, 19 ; XLII, 6 ; II, 12 ; XVI, 15 ; VII, 21 ; X, 9 ; XVII, 16 ; XXI, 26. Traduction Édouard Dhorme, La Bible. Ancien Testament, Paris, Gallimard, Bibliothèque de la Pléiade, tome II, 1959.

18. «Si je vais à l'orient, il n'y est pas, / et à l'occident, je ne l'aperçois pas ; / au nord je l'ai cherché et je ne l'ai point vu, / je reviens au midi et je ne le vois pas davantage ! »XXIII, 8-9).

19. La Bible. Ancien Testament, op. cit., note p. 1310.

20. Emmanuel Levinas, Totalité et Infini, La Haye, Martinus Nijhoff, 1974, p. 168-169.

21. Emmanuel Levinas, ibid., p. 74-75. Voir aussi Maurice Blanchot, «La parole plurielle», in L'Entretien infini, Paris, Gallimard, coll. «Blanche », 1969, p. 5 : «Or, connaître par la mesure de l'“inconnu", aller à la familiarité des choses en en réservant l'étrangeté, se rapporter à tout par l'expérience même de l'interruption des rapports, ce n'est rien d'autre qu'entendre parler et apprendre à parler. »

\section{RÉSUMÉS}

La chute de cheval de Montaigne, l'attaque de Rousseau par un chien, le vacillement de Proust sur les pavés sont autant d'accidents ouvrant à la création. L'expérience de la souffrance, de la perte, de la destruction permet de comprendre l'activité poiétique comme passage de l'être au non être, commerce avec l'inexistant. Penser la création à partir des plaies de la sensibilité et de leur cicatrisation conduit à repenser le statut de la liberté (ou nécessité) créatrice ainsi que les conditions temporelles de l'émergence des œuvres, qui adviennent toujours sur le fond d'une altérité.

\section{INDEX}

Mots-clés : poiétique, mémoire, trace, souffrance, accident, événement, commencement, altérité 
AUTEUR

DANIEL PAYOT

Professeur de philosophie de l'art à la Faculté des Arts de l'université de Strasbourg, directeur de l'équipe d'accueil EA 3402 «Approches contemporaines de la création et de la réflexion artistique ». 